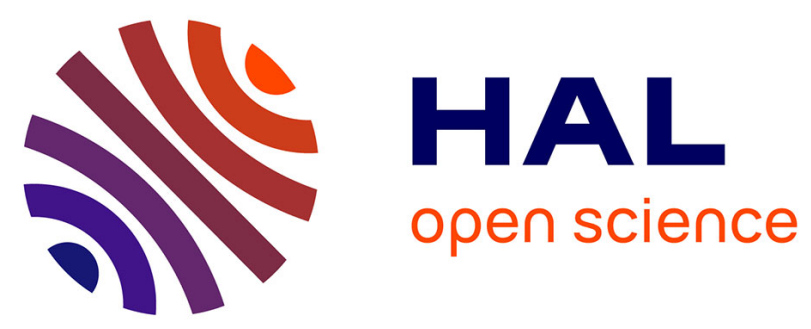

\title{
Du BoP dans le beat, une analyse des transformations numériques dans la musique
}

\author{
Robin Charbonnier, Pierre Poinsignon, Thomas Paris
}

\section{To cite this version:}

Robin Charbonnier, Pierre Poinsignon, Thomas Paris. Du BoP dans le beat, une analyse des transformations numériques dans la musique. Revue Française de Gestion, 2021, 47 (294), pp.115-134. 10.3166/rfg.2021.00502 . hal-03328239

\section{HAL Id: hal-03328239 \\ https://hal.science/hal-03328239}

Submitted on 29 Aug 2021

HAL is a multi-disciplinary open access archive for the deposit and dissemination of scientific research documents, whether they are published or not. The documents may come from teaching and research institutions in France or abroad, or from public or private research centers.
L'archive ouverte pluridisciplinaire HAL, est destinée au dépôt et à la diffusion de documents scientifiques de niveau recherche, publiés ou non, émanant des établissements d'enseignement et de recherche français ou étrangers, des laboratoires publics ou privés. 


\section{Du BoP dans le beat ; une lecture Bas de la Pyramide des transformations numériques de l'industrie de la musique}

\section{Résumé :}

Dans un marché de la musique morne, le distributeur numérique Believe s'est développé au point de concurrencer les Majors du secteur. Cet article analyse ses choix stratégiques singuliers pour en tirer des enseignements sur les mutations numériques. L'ouverture de Believe à des artistes exclus de l'industrie nous conduit à mobiliser la littérature «Bas de la Pyramide ». Nous montrons que ce choix est à l'origine d'un modèle qui casse les frontières traditionnelles de l'industrie en mêlant prestation technique pour le plus grand nombre et accompagnement d'artistes sélectionnés.

\section{Mots-clefs :}

Bas de la Pyramide, industries créatives, créativité, musique, numérisation

\section{Abstract:}

In a struggling music market, the digital distributor Believe has grown to the point of competing with the Majors in the industry. This article analyzes its singular strategic choices, to draw lessons on digital mutations. The extension of Believe to artists excluded from the industry leads us to call upon the "Bottom of the Pyramid" literature. We show that this choice is at the origin of a model that breaks the traditional boundaries of the industry by mixing technical services for the greatest number of people and support for selected artists.

Keywords:

Bottom of the Pyramid, creative industries, creativity, music, digitization

Biographies des auteurs :

Doctorant à i3 CRG-École polytechnique, Robin Charbonnier est un ancien élève d'HEC Paris où il a étudié le management de l'art et de la création. Son travail de doctorat, mené dans le cadre d'un contrat CIFRE au Centre National de la Musique (CNM), porte sur la structuration de la filière musicale et la prise en compte de la diversité culturelle.

Doctorant à i3 CRG-École polytechnique, Pierre Poinsignon est un ancien élève d'HEC Paris où il a étudié le management de l'art et de la création. Son travail de doctorat porte sur l'innovation radicale dans les mondes de l'art, à travers quatre terrains dans les industries du cinéma, de l'édition, de la musique et du jeu vidéo.

Thomas Paris est chercheur au CNRS (GREGHEC) et professeur affilié à HEC Paris. Docteur en gestion, il s'est spécialisé sur l'économie de la création et des industries créatives. Il étudie ces secteurs (cinéma et audiovisuel, musique, mode, édition, architecture, jeu vidéo, grande cuisine, design...) sous les angles managérial, organisationnel et sectoriel, en collaboration avec leurs acteurs. Il travaille par ailleurs sur le management de l'innovation, l'entrepreneuriat, l'économie numérique et les politiques culturelles. 


\section{INTRODUCTION}

Au tournant des années 2000, le marché de la musique enregistrée atteint son apogée ; c'est l'âge d'or du disque. L'industrie musicale a pourtant déjà entamé une mutation déclenchée par les nouvelles pratiques numériques qui ont émergé au milieu des années 1990 : les échanges illégaux de contenus donnent lieu au contournement des circuits traditionnels du marché. Cette nouvelle donne numérique participe fortement au déclin de l'industrie du disque (Bourreau, Labarthe-Piol, 2006) : les ventes de musique enregistrée en France passent de 1,4 milliard d'euros en 2002 à 515 millions d'euros en 2015 (SNEP, 2019). La filière semble pourtant garder ses schémas d'organisation (Beuscart, 2007), à l'instar des acteurs majeurs de l'industrie du disque qui ont maintenu leurs choix malthusiens dans l'investissement artistique et tâtonné face à la révolution numérique (Moyon, 2011).

L'exclusion d'une large partie des aspirants à la création est en effet une caractéristique du fonctionnement des industries culturelles et créatives (Menger, 1999 ; Caves, 2000). Et alors que le numérique suggère la possibilité d'un nouvel ordre où la relégation de certains pourrait être largement remise en cause (Anderson, 2006), cela n'est pas confirmé par les faits (Benghozi, Benhamou, 2008). Pourtant, dans d'autres secteurs, les stratégies orientées vers les pans de marché délaissés ont commencé à avoir le vent en poupe précisément à cette période : la littérature sur le Bas de la Pyramide, ou « BoP » pour «Bottom of the Pyramid» (Prahalad, Lieberthald, 1998 ; Prahalad, Hammond, 2002 ; Prahalad, Hart, 2002 ; Prahalad, 2004), montre la possibilité de développer des offres rentables pour des populations exclues. Dès lors, qu'est-ce qui empêche le développement d'une telle stratégie dans un champ comme celui de la musique? Plus généralement, quels sont les facteurs qui favorisent le développement de telles stratégies?

Pour y répondre, nous nous intéressons au cas d'une entreprise fondée en 2005 qui a mené une stratégie à contre-courant de celles alors menées dans le secteur de la musique enregistrée et qui, face à un marché peu dynamique, a connu une croissance remarquable pour atteindre un chiffre d'affaires d'environ 700 millions d'euros en 2019. Believe, à l'inverse des acteurs historiques, distribue plusieurs centaines de milliers d'artistes, fonde son modèle économique sur des acteurs considérés comme peu profitables et fait partie des quelques nouveaux entrants parvenant à tirer rapidement profit des changements technologiques.

En nous appuyant sur les travaux sur les industries culturelles et créatives d'une part et sur la littérature BoP d'autre part, nous étudions le fonctionnement et le succès de l'entreprise, pour analyser son modèle, comprendre dans quelle mesure il s'apparente à une stratégie BoP, pour enrichir la compréhension de la transformation numérique dans les industries culturelles d'un côté, et les conditions de développement des stratégies BoP de l'autre.

\section{REVUE DE LITTÉRATURE}

Notre questionnement associe deux courants de recherche distincts : les industries créatives et culturelles d'un côté, les stratégies BoP de l'autre; cette dernière littérature servant de grille de lecture pour le cas.

\subsection{Les promesses non tenues du numérique dans l'industrie de la musique}

L'industrie de la musique appartient aux industries créatives, un ensemble de secteurs culturels qui partagent des spécificités en matière d'économie, d'organisation et de gestion 
(DeFillippi, Grabher et Jones, 2007). La surabondance de candidats et le rationnement des positions y sont des traits structurels (Menger, 1999), donnant lieu à des marchés marqués par une différenciation verticale des talents (Caves, 2000) entre les talents de la liste-A («A-list $»)$, éléments non-substituables au sein d'un projet, et les talents de la liste-B («B-list»), éléments participant à la production créative, mais substituables. Par ailleurs, la présence de gardes-barrières («gatekeepers »), des acteurs qui jouent un rôle dans l'accès des propositions artistiques au marché, se traduit aussi par des inégalités d'accès des talents aux marchés.

Comme beaucoup de secteurs culturels, l'industrie de la musique enregistrée est organisée autour de ces logiques, dans une structure d'oligopole à franges : le marché est historiquement dominé par quelques Majors et le reste du marché est composé d'une multitude de petites structures, notamment des labels. Une structure sous-tendue par un star system (Benhamou, 2002) marqué par de fortes inégalités entre artistes.

Cette dernière notion est à la base de la théorie de la longue traîne (Anderson, 2006) : la structure de consommation des industries culturelles se concentre sur des artistes stars, et un pan entier de la production se retrouve négligé par la consommation. Au tournant des années 2000, l'avènement des technologiques numériques stimule la circulation des biens de type informationnel, dont les coûts de reproduction sont très faibles et dont les infrastructures technologiques facilitent la distribution (Varian, Shapiro, 1999); la théorie d'Anderson inférait que ce basculement dans le monde numérique permettrait à des acteurs de réduire leurs coûts et d'exploiter la longue traîne tout en en tirant profit. Anderson prend notamment le cas d'Amazon, qui, exploitant la longue traîne, aurait développé une stratégie plus rentable qu'une traditionnelle stratégie de vente de blockbusters.

Dans la musique, ces technologies numériques, en plus de nouveaux moyens d'écoute, ont permis non seulement à la pratique musicale et aux moyens de production de se démocratiser (Bacache, Bourreau, Gensollen, Moreau, 2009), mais aussi à une offre légale en ligne de prendre son essor au début des années 2010, fondée sur une logique d'accès (Rifkin, 2000) et proposée par les plateformes de streaming musical. Pour le secteur, ces transformations étaient la perspective d'une remise en cause des goulots d'étranglements qui façonnent l'industrie (Benghozi, Paris, 2001) et de la fin de l'intermédiation, notamment de la mainmise des gardes-barrières constitués par les maisons de disque.

Cependant, du point de vue de la consommation, la théorie de la longue traîne n'a pas été confirmée par les faits : le basculement vers le numérique n'a pas entraîné de lissage de la consommation et les best-sellers musicaux ont été renforcés (Benghozi, Benhamou, 2008). De fait, la théorie de la longue traîne nie la dimension collective de la consommation, notamment la construction sociale de la valeur des œuvres (Becker, 1982), ainsi que le rôle des «dispositifs de jugements» (Karpik, 2007) comme les prescripteurs qui créent un phénomène d'accentuation.

Du point de vue de l'offre, la capacité des entreprises à développer un modèle qui exploite la longue traîne n'a pas été confirmée. Le cas de Believe est à cet égard intéressant. Dans un secteur qui génère toujours des exclus, l'entreprise se présente comme l'opposée d'un gardebarrière, travaillant avec tous les artistes qui le souhaitent, et affichant un succès qui semble au premier abord reposer sur ce choix, et donc sur l'exploitation de la longue traîne. Cette capacité singulière à exploiter un segment composé d'acteurs a priori peu rentables mais en nombre important nous incite à mobiliser la littérature des stratégies BoP. 


\subsection{Les stratégies BoP}

La théorie «Bottom of the Pyramid» vise à démontrer qu'il y a possibilité pour les grandes entreprises multinationales de faire du profit, tout en concourant à la réduction de la pauvreté, en abordant des marchés latents composés de consommateurs aux revenus les moins élevés, compensés par leur nombre important (Guesalaga, Marshall, 2008). Une stratégie BoP consiste à revoir son offre en proposant des modèles d'affaires et une chaîne de valeur adaptés à ce segment de consommateurs (London, Hart, 2004).

Cette théorie originelle a donné lieu à des remises en cause (Pitta, Guesalagua, Marshall, 2008), qui relèvent notamment que, du fait de calculs et de définitions contestables du seuil de pauvreté (Karnani, 2007 ; Kolk, Rivera-Santos, Rufín, 2014), le potentiel économique des marchés du Bas de la Pyramide serait surestimé et la rentabilité de la stratégie BoP serait en fait discutable pour les acteurs privés internationaux (Karnani, Garrette, Kassalow, Lee, 2010 ; Pitta, Guesalagua, Marshall, 2008).

Des travaux successifs ont affiné la théorie. En premier lieu, ils ont reconsidéré les acteurs impliqués dans cette stratégie ainsi que leur caractérisation, montrant que les multinationales n'étaient pas les principales instigatrices des projets BoP et que, lorsqu'elles l'étaient, elles se retrouvaient insérées dans des logiques locales modifiant une stratégie économique qui ne peut se départir des dimensions sociale, environnementale et politique (Cholez, Trompette, Vinck, Reverdy, 2010). Ils ont par ailleurs observé que le consommateur pauvre, figure phare de la théorie, pouvait aussi être envisagé comme participant pleinement au renouveau des chaînes de valeur en étant par exemple lui-même producteur (Karnani, 2007), et non plus seulement un simple bénéficiaire de produits existants (Kolk, Rivera-Santos, Rufín, 2014).

Les modèles qui en découlent (Anderson, Billou, 2007 ; Gollakota, Gupta, Bork, 2010 ; Martinet, Payaud, 2010 ; Schrader, Freimann, Seuring, 2012) et qui permettent d'analyser des stratégies BoP, restent conçus à l'attention des entreprises capitalistes (Dumalanède, 2017).

Le succès de Believe interpelle car il s'est construit rapidement dans un secteur en difficulté. En première approche, l'on peut être tenté d'expliquer ce succès par une maîtrise exceptionnelle du marketing numérique, ou par une faculté de détection des nouveaux talents, mais aucune de ces explications ne paraît suffisante au regard du développement exceptionnel de l'entreprise, parfois qualifiée de licorne ou de quatrième Major de la musique.

La théorie de la longue traîne dans les industries créatives, aussi bien que la théorie BoP ont chacune mis en avant la possibilité de développer des stratégies qui exploitent des pans de marché inexploités, sur des arguments assez proches de rentabilisation sur les volumes. Or, dans l'industrie de la musique, cette ouverture n'a pas été observée dans un premier temps, l'industrie conservant ses goulots d'étranglement et son hyper-concentration. Les travaux récents sur la théorie BoP soulignent notamment un élargissement de la caractérisation du bas de la pyramide, dépassant la première définition de population pauvre. Les artistes exclus de l'industrie de la musique pouvant ainsi être qualifiés de bas de la pyramide, pourquoi les grandes entreprises traditionnelles n'ont-elles pas développé des stratégies pour cette population représentant un marché non exploité et pourquoi cela est-il venu d'un acteur extérieur? Cela nous conduit à interroger les conditions d'apparition de stratégies BoP. Si la littérature a ouvert la possibilité de ces stratégies à des entreprises et à des marchés diversifiés, qu'est-ce qui peut expliquer leur développement ou leur absence de développement dans un secteur donné ? C'est à cette question que nous souhaitons apporter des éléments de réponses par l'analyse du cas de Believe. 


\section{Méthodologie}

Notre intérêt pour Believe a émergé de la singularité de l'entreprise dans l'industrie musicale, qui affichait une forte croissance dans un secteur morne et des choix éditoriaux et stratégiques disruptifs, couronnés de succès dans les charts musicaux. Cette singularité nous a conduits à faire reposer notre recherche sur une étude de cas unique (Yin, 2012).

Sans préjuger des questions à traiter et pour appréhender ce terrain nouveau, nous avons adopté une approche abductive (Dumez, 2016) en deux temps : une phase exploratoire s'appuyant sur un recueil des témoignages des employés, avec l'objectif de comprendre le fonctionnement, la structure et les processus de l'entreprise, et mettre au jour les raisons de sa réussite ; puis un temps d'allers-retours entre le matériau - d'autres entretiens - et l'analyse, ayant conduit à retenir la littérature $\mathrm{BoP}$ comme grille de lecture pertinente.

La recherche s'appuie sur 19 entretiens semi-directifs de 45 à 120 minutes (dont 16 enregistrés et retranscrits), entre octobre 2019 et février 2020, avec des représentants des différents métiers de Believe, des artistes et des plateformes de distribution. Les grilles d'entretien, dans une approche compréhensive, visaient à mettre au jour les tâches, les relations et les outils de chacun dans l'environnement étudié (Becker, 1998). Ce matériau a été codé en fonction de catégories ayant émergé à la fois du terrain et de la littérature. À titre d'exemple : parmi nos quatre codes de premier niveau, le code 2 était consacré à la stratégie de l'entreprise; il se subdivisait en sous-niveaux, notamment le code 2.1 consacré à la technologie, le code 2.2 consacré aux musiques urbaines ou encore le code 2.3 sur l'organisation pyramidale assimilable à une stratégie BoP.

\section{AUX ORIGINES DE LA STRATEGIE DE BELIEVE}

Les résultats sont présentés en suivant l'histoire de la création et de la structuration de Believe, afin de comprendre les choix stratégiques qui ont été opérés.

\subsection{L'opportunité d'une spécialisation dans la distribution numérique}

La pénétration du numérique dans l'industrie musicale au tournant des années 2000 laisse entrevoir la transformation des canaux de distribution, et la transition vers une distribution numérique. En 2005, Believe - à l'origine Believe Digital - se crée sur la conviction que l'apparition inéluctable d'un marché de la distribution numérique générera un appel d'air sur des fonctions techniques.

L'entreprise naît ainsi comme un intermédiaire technique entre les ayants droit (maisons de disques, labels indépendants, artistes autoproduits...), qui cherchent à exploiter leurs catalogues de titres dans l'environnement numérique, et les acteurs de la distribution numérique (les magasins numériques tels les sites de la Fnac, de Virgin, iTunes, puis les plateformes de streaming comme Spotify et Deezer). Believe débute en démarchant des producteurs et des artistes pour tenter de les convaincre de numériser leurs catalogues et ainsi monétiser leurs contenus sur le numérique ; en parallèle, elle noue des contrats de distribution avec les boutiques numériques (les stores) à la recherche d'agrégateurs de contenus.

Le métier de Believe s'articule alors autour de deux flux d'activités. L'entreprise réceptionne les enregistrements des créateurs, les convertit en format numérique, effectue un travail 
d'association et de vérification des métadonnées du contenu pour être capable de fournir rapidement aux stores le titre du morceau, le nom de l'auteur, la pochette, ou encore l'identification de paroles sensibles. Une fois ce formatage opéré, l'entreprise effectue la livraison aux magasins de musique en ligne, au format numérique choisi par chacun d'eux.

Après la mise à disposition de la musique et sa consommation sous forme d'écoute ou de téléchargement, s'enclenche une nouvelle étape : Believe se charge de réclamer, collecter et répartir les royautés dues au producteur. En complément, les données de consommation sont mises à disposition des producteurs ou artistes sous forme de tableaux de chiffres permettant des suivis de performance et d'activité.

\section{Figure 1. Le rôle d'intermédiaire numérique de Believe Digital dans l'industrie de la musique}

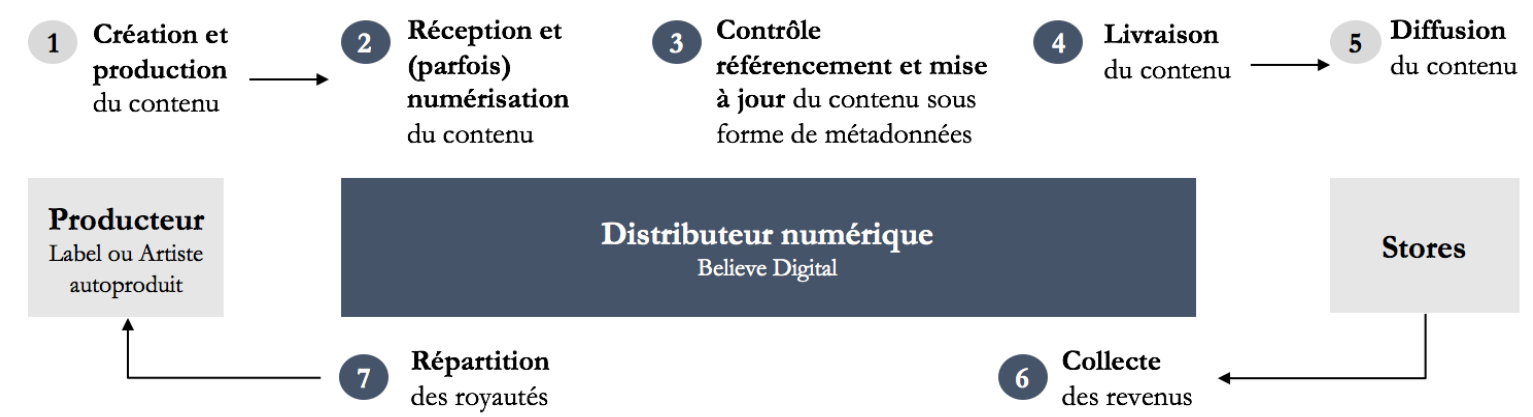

Ce positionnement premier d'intermédiaire technologique se lit aussi dans le fait que Believe effectue ses principaux investissements dans le développement d'outils en interne : dès 2005, un instrument de récupération et de publication des données de consommation est créé. Ce logiciel, développé en interne, appelé Backstage, centralise toute l'information des contenus de chaque producteur lié à Believe.

Chef du Département Produit : " Nous, on a toujours développé nous-mêmes, on a toujours eu des équipes de développeurs, et le PDG a toujours eu une vision très technologique de la boîte. À la différence des majors qui ont beaucoup de mal à franchir le cap de la digitalisation et de la numérisation de la consommation de la musique, nous c'était notre cœur de métier, notre fonds de commerce. »

Figure 2. La construction du modèle à partir de $2005^{1}$

\footnotetext{
${ }^{1}$ Nous représentons le marché des artistes sous forme de trois grandes catégories, en nous appuyant sur la terminologie de Caves (A-List/B-List) et en introduisant la catégorie des artistes préalablement exclus du marché («Bas de la pyramide»). Nous sommes conscients que cette classification ne renvoie pas exactement aux définitions de Caves, mais nous les mobilisons, par souci de clarté, pour caractériser les artistes en fonction de leur importance pour les labels.
} 


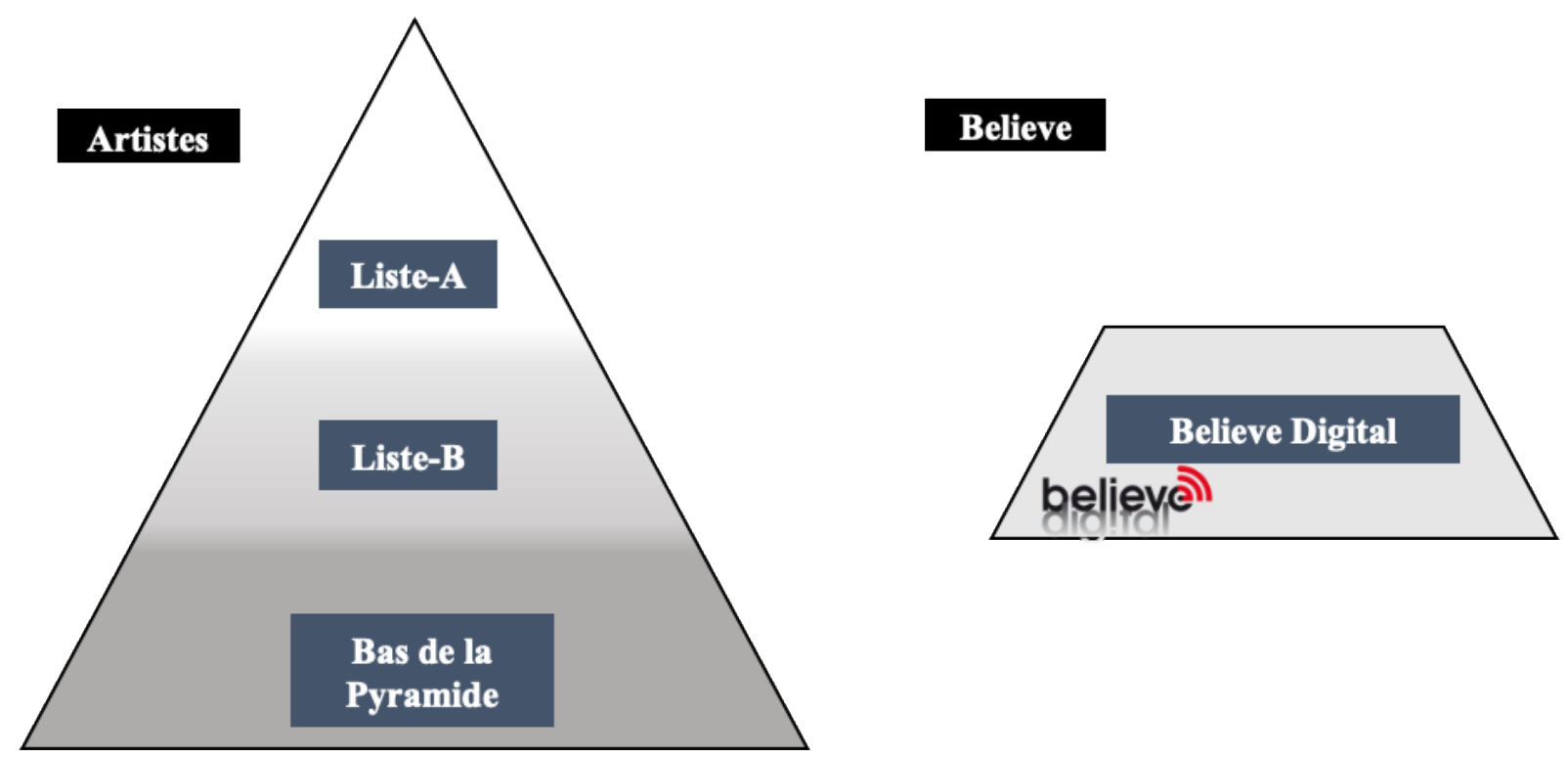

\subsection{Le développement d'une offre pour les artistes délaissés par l'industrie}

Positionnée entre les producteurs et les plateformes de distribution, témoignant d'une volonté croissante d'accélérer et d'automatiser ses processus de transfert des contenus, développant une capacité technologique à traiter des volumes, Believe se place rapidement comme un acteur de référence sur le marché naissant de la distribution numérique. Des capacités qui lui permettent, contrairement aux Majors, de s'adresser à tout type de créateur de contenu désireux de le voir distribué.

Jusqu'en 2015, Believe s'attache ainsi à proposer deux offres, majoritairement destinées aux artistes exclus des champs d'action des acteurs traditionnels de l'industrie : des artistes non « signés » - c'est-à-dire n'ayant pas de contrat avec un label ou un distributeur, comme les artistes entrant dans le métier, les artistes amateurs, les artistes autoproduits et certains artistes confirmés mais aux contrats rendus par les Majors -, des artistes de petits labels indépendants, ou des artistes au genre musical peu médiatisé. Pour beaucoup, des artistes souhaitant être leur propre producteur mais frustrés par les barrières dressées.

La première, proposée à tous les artistes, est une offre simplifiée et exclusivement numérique, donc particulièrement adaptée aux artistes autoproduits ou entrant dans le métier. Elle prend forme en 2009 avec la création de Zimbalam, une plateforme d'auto-distribution en ligne où les producteurs et créateurs peuvent facilement déposer leurs enregistrements et les voir distribuer sur les stores de leur choix. L'artiste récupère l'intégralité de ses royautés, mais doit payer un forfait fixe qui constitue la rémunération de Believe. Fin 2014, Zimbalam fusionne avec Tunecore, le concurrent américain que Believe vient de racheter.

Direction : «On a commencé à attaquer le segment des artistes un peu plus petits et celui des artistes en développement parce que sur ces marchés-là, l'élément clé de l'offre, c'est la capacité à utiliser la technologie pour gérer des volumes. »

Le deuxième étage de l'offre se construit sur les services historiques de distribution numérique, enrichis de services marketing, que Believe propose en contrepartie d'un pourcentage pris sur les revenus. Ils forment la division Believe Distribution. Avec cette offre, 
Believe se concentre sur des projets plus prometteurs, choisis, sur lesquels elle investit plus de moyens.

Ainsi, Believe a développé en parallèle deux offres, l'une dédiée au marché existant des artistes signés, l'autre, novatrice, pour des artistes qui ne bénéficient pas de contrat avec un label. Le volume d'artistes traités est bien plus important pour ce second marché, que nous appelons ici «bas de la pyramide».

Figure 3. La construction du modèle à partir de 2009

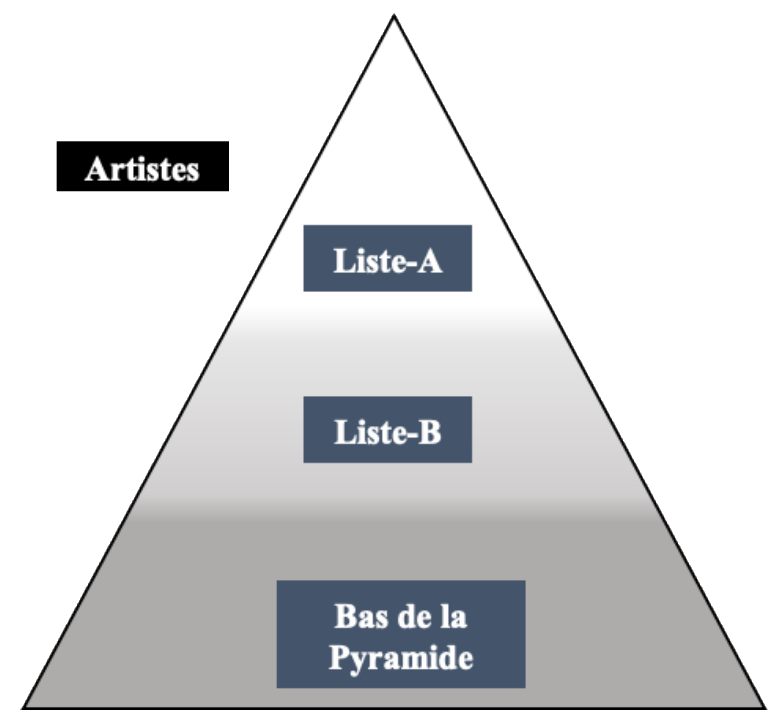

Believe

\subsection{L'augmentation des volumes par l'internationalisation}

Après la différenciation des services auprès des artistes et producteurs, ce sont les stratégies des plateformes, les autres clients de Believe, qui influent sur son orientation stratégique.

En 2012, Apple cherche des partenaires pour mener l'internationalisation de son offre musicale iTunes. Apple agrégeait jusqu'alors elle-même le contenu musical, en intégrant des services, processus et outils similaires à Believe Digital. Mais en 2012, Apple lance une internationalisation de sa plateforme iTunes dans plus de cinquante pays, ce qui l'amène à recentrer son activité sur le versant des audiences plutôt que sur celui des fournisseurs de contenu, dont la gestion représente une charge technique et juridique conséquente. Sur la base d'un audit, Apple sélectionne des entreprises capables d'assurer la livraison d'un contenu de qualité et de garantir la chaîne des droits liés aux enregistrements (voir Figure 1). Trois agrégateurs de musique sont retenus pour alimenter iTunes dans le monde : The Orchard (qui sera racheté en 2015 par Sony), Believe et Tunecore (qui sera donc racheté par Believe fin 2014).

La mise à l'écart des concurrents crée un élan pour Believe. Tous les fournisseurs de contenus d'Apple sont redirigés vers Believe, ce qui se traduit par un accroissement notable des catalogues à traiter. Sa panoplie de services et de technologies (cf. Tableau 1) permet à l'entreprise d'absorber ces volumes. Ses outils, avec Backstage au premier rang, sont répliqués dans les différents territoires et permettent de réaliser d'importantes économies d'échelles. 
Direction : "Quand on développe une plateforme techno, peu importe qu'on la développe pour distribuer et numériser des $\mathrm{CD}$ italiens ou espagnols, on est déjà intégrés numériquement avec Apple, donc on a tout intérêt à être capable de ramener des CD et de la musique de l'intégralité des pays du monde, on va utiliser la même plateforme techno et on va avoir des effets d'échelle très forts sur la techno. »

Devenu partenaire officiel d'Apple, Believe fournira, en 2019, plus de $30 \%$ des volumes de musique mondiaux de la plateforme, signe du changement d'échelle qu'a entrainé cette consolidation.

Tableau 1 : Les outils technologiques de Believe en 2019

\begin{tabular}{|c|c|}
\hline Outil & Description \\
\hline \multicolumn{2}{|r|}{ Signature et proposition commerciale } \\
\hline Zimbalam & Plateforme de livraison aux stores \\
\hline Backstage & Suivi des statistiques des comptes émergents et prometteurs \\
\hline PipeDrive & $\begin{array}{l}\text { Suivi des actions commerciales des employés, référencement des artistes } \\
\text { suivis à qui Believe souhaite faire une proposition commerciale }\end{array}$ \\
\hline \multicolumn{2}{|l|}{ Fiches d'avance } \\
\hline $\begin{array}{r}\text { dont Tableau de } \\
\text { projection de } \\
\text { revenus }\end{array}$ & Permet le placement sur la typologie d'artiste et le calcul de l'avance \\
\hline $\begin{array}{r}\text { dont Typologie } \\
\text { d'artistes }\end{array}$ & $\begin{array}{l}\text { Permet de proposer l'offre de services correspondant à chaque typologie } \\
\text { d'artistes }\end{array}$ \\
\hline \multicolumn{2}{|r|}{ Gestion et promotion des catalogues } \\
\hline Backstage & Création des sorties, versement des royalties et analyse de données \\
\hline $\begin{array}{l}\text { Système de } \\
\text { Ticketing }\end{array}$ & $\begin{array}{l}\text { Mise en forme et mise à jour des données, assistance aux problèmes } \\
\text { clients }\end{array}$ \\
\hline Zimbalam & Plateforme de livraison aux stores \\
\hline Business Manager & $\begin{array}{l}\text { Affiche les Analytics des réseaux et notamment degré de conversion des } \\
\text { actions de marketing digital (taux de répétition, taux de clics...) }\end{array}$ \\
\hline Newsletter & $\begin{array}{l}\text { Production de newsletters (import des contacts, templates, analyse de la } \\
\text { portée de la newsletter...) }\end{array}$ \\
\hline Backlink & $\begin{array}{l}\text { Communication digitale de liens sécurisés pour faire écouter aux pros, } \\
\text { permettant de faire des campagnes dédiées }\end{array}$ \\
\hline $\begin{array}{l}\text { Tableaux des } \\
\text { sorties }\end{array}$ & $\begin{array}{l}\text { Tableaux de pitch des sorties à destination des éditorialistes des } \\
\text { plateformes, avec des ordres de priorité }\end{array}$ \\
\hline \multicolumn{2}{|r|}{ Évaluation de la performance des comptes } \\
\hline Fichiers Excel & Notation de la rentabilité des projets \\
\hline
\end{tabular}

\subsection{La construction d'un catalogue et la nécessité d'avoir des artistes stars : la montée en gamme de Believe}

À partir de 2015, Believe entame une série de rachats qui vise à lui permettre de disposer des ressources liées aux services pour les artistes établis, autrement dit les services proposés par les Majors et principaux labels. Believe rachète Naïve et Musicast en 2016, deux structures avec des lignes éditoriales fortes respectivement sur les musiques savantes et sur le rap. Puis en 2017, une partie des catalogues de Warner est rachetée. La même année, Believe Rec - 
label créé en 2011 pour se donner les moyens de signer les artistes - prend un virage affirmé vers les musiques urbaines en devenant AllPoints, tandis que le label Animal 63 est créé en association avec Savoir Faire. Ils forment alors la partie « labels » de Believe, soit le troisième étage de services aux artistes.

Directeur Marketing: «Et sur cette base se sont ensuite rajoutées les briques traditionnelles du marché de la musique, ressemblant plus à une maison de disque : avoir un label, signer des contrats d'artiste et de licence, avoir une distribution physique, avoir une capacité à attirer des seniors du marché, des gens (...) qui ont eu une carrière en Major, un peu plus issus de l'ancien monde moins digitalisé, et ce sont ces gens-là qui ont structuré la façon d'aller parler au marché de la musique. »

L'activité « labels » rassemble les artistes prioritaires, ceux sur lesquels Believe investira plus de moyens que sur les artistes de Believe Distribution ou Tunecore. Les artistes en label disposent d'une équipe dédiée, comme pour Believe Distribution, mais cette équipe est plus nombreuse et focalisée sur un nombre restreint de projets. L'ensemble de ces activités personnalisées relèvent donc d'un travail plus artisanal qu'industriel.

Cette évolution de l'entreprise vers l'artistique, au sens où elle intègre des choix éditoriaux, en complément de la base technologique, permet désormais d'offrir des services aux artistes confirmés, cible traditionnelle des Majors.

Figure 1. L'architecture du modèle à partir de 2015
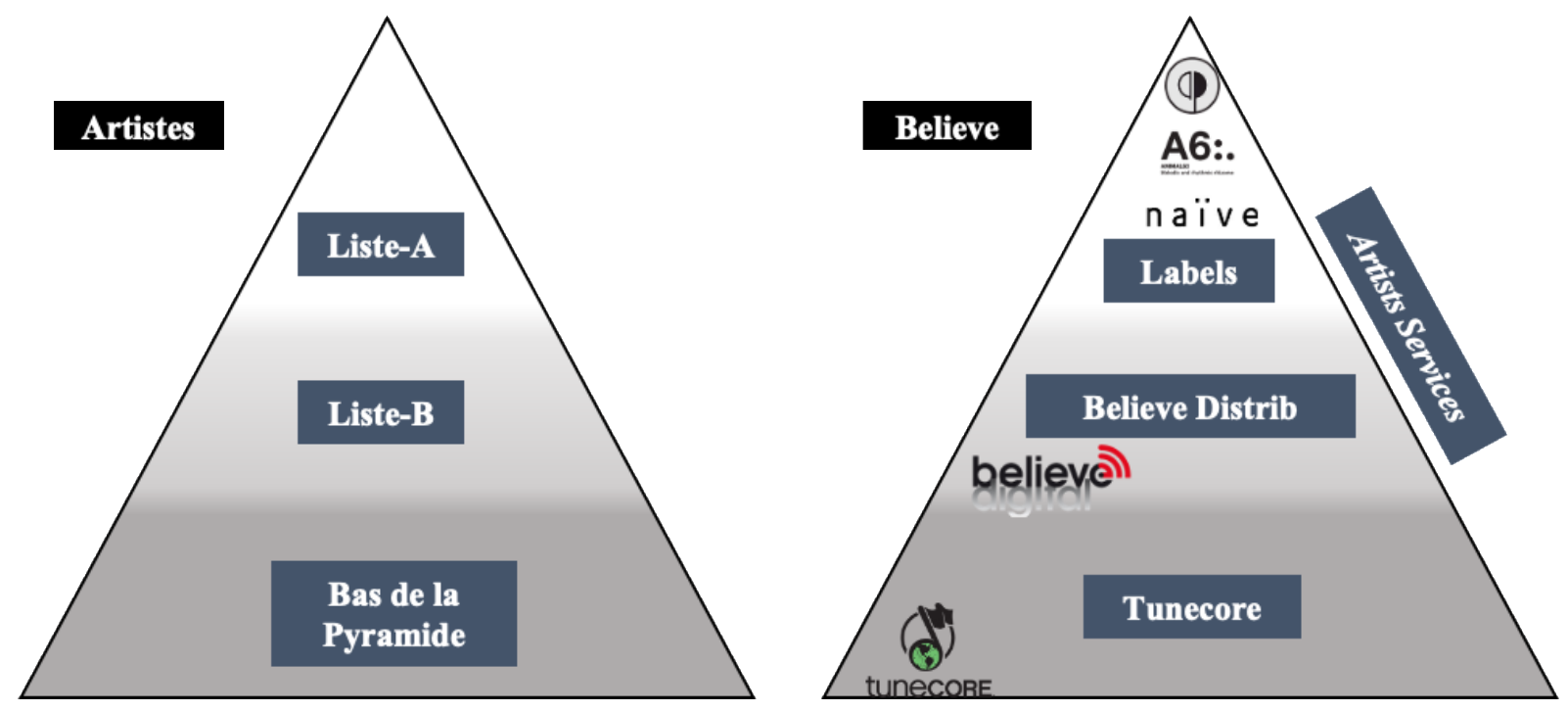

\subsection{Une offre au spectre large, fonction d'une segmentation pyramidale}

En, 2019, Believe est structurée en trois divisions dont chacune dispose de ses propres équipes, ses propres offres et ses propres outils (parfois partagés), dans une organisation pyramidale: Believe Label propose un service très complet à un petit nombre d'artistes, TuneCore propose des services à plus faible valeur ajoutée à un grand nombre d'artistes et Believe Distribution se situe entre les deux.

Direction : «On s'est dit que dans la musique, il y a trois segments de marché : le segment de marché des artistes amateurs (...), le segment des artistes de taille moyenne et le segment des top artists. Et pour être capable d'avoir $20 \%$ de parts de marché on y 
arrivera beaucoup plus vite si on est capables d'intervenir et d'être un acteur important sur les trois segments de marché. »

Par conséquent, le Bas de la Pyramide repose sur un volume de signatures important mais une marge faible pour Believe, et plus on monte dans les étages de l'organisation, plus Believe prélève une part élevée sur les revenus générés par le projet musical.

Fin 2019, une quatrième section transversale distincte prend de l'ampleur: les Artists Services. Elle pioche dans la gamme élargie des services du groupe et s'appuie sur l'introduction d'une plus grande latitude dans la constitution des contrats. Traditionnellement, les maisons de disques proposaient un contrat de distribution, un contrat de licence ou un contrat d'artiste. Avec Artists Services, Believe propose différents types de «contrats de distribution améliorée » qui reprennent une répartition des recettes proche du contrat de distribution mais en y adossant plus de services, notamment celui du trademarketing. Ce large éventail de services et leur adaptabilité dans le contenu et dans le temps renforcent l'accessibilité de l'offre aux artistes et permettent une grande mobilité des artistes au sein de l'organisation : ils peuvent y faire tout leur parcours.

Tableau 2 : Les différents niveaux de services en fonction des artistes en 2019 


\begin{tabular}{|c|c|c|c|}
\hline & TuneCore & Believe Distrib & $\begin{array}{c}\text { Believe Labels et Artists } \\
\text { Services }\end{array}$ \\
\hline $\begin{array}{l}\text { Type d'artistes en } \\
\text { contrat }\end{array}$ & $\begin{array}{l}\text { Groupe hétérogène : amateurs, } \\
\text { artistes entrants dans } \\
\text { l'écosystème, artistes de taille } \\
\text { moyenne et même artistes } \\
\text { structurés }\end{array}$ & $\begin{array}{l}\text { Artistes de taille moyenne mais } \\
\text { aussi parfois confirmés }\end{array}$ & $\begin{array}{l}\text { Artistes confirmés ou } \\
\text { prometteurs }\end{array}$ \\
\hline $\begin{array}{l}\text { Type de contrats } \\
\text { proposés }\end{array}$ & $\begin{array}{l}\text { Souscription aux conditions de } \\
\text { la plateforme }\end{array}$ & $\begin{array}{l}\text { Contrats de distribution } \\
\text { standard }\end{array}$ & $\begin{array}{l}\text { Contrats Artists Services } \\
\text { (distribution améliorée) et } \\
\text { Artists Development (contrats } \\
\text { d'artiste et contrats de licence) }\end{array}$ \\
\hline $\begin{array}{l}\text { Degré } \\
\text { d'automatisation des } \\
\text { services }\end{array}$ & $\begin{array}{l}\text { Services automatisés } \\
\text { uniquement }\end{array}$ & $\begin{array}{c}\text { Services automatisés et métiers } \\
\text { artisanaux }\end{array}$ & $\begin{array}{l}\text { Services automatisés et } \\
\text { métiers artisanaux }\end{array}$ \\
\hline Services disponibles & Distribution numérique & $\begin{array}{c}\text { Distribution } \\
\text { numérique } \\
\text { Label Manager } \\
\text { Trademarketing } \\
\text { Financement partiel du projet } \\
\text { avec avance remboursable } \\
\text { Conseils informels }\end{array}$ & $\begin{array}{c}\text { Distribution numérique } \\
\text { Chef de projet } \\
\text { Trademarketing } \\
\text { Financement complet du projet } \\
\text { avec avance non-remboursable } \\
\text { possible } \\
\text { Marketing et promotion } \\
\text { Réseau professionnel } \\
\text { Direction artistique } \\
\text { Distribution physique } \\
\text { Publishing } \\
\text { Tour } \\
\text { Synchronisation } \\
\text { Influencing } \\
\text { Subventions } \\
\text { Gestion des droits voisins }\end{array}$ \\
\hline $\begin{array}{l}\text { Nombre d'artistes en } \\
\text { contrat }\end{array}$ & $\begin{array}{l}\text { Plusieurs centaines de milliers } \\
\text { d'abonnés annuels }\end{array}$ & $\begin{array}{l}\text { Plusieurs milliers de comptes } \\
\text { en France }\end{array}$ & $\begin{array}{l}\text { Plusieurs centaines de } \\
\text { comptes en France }\end{array}$ \\
\hline Marges pour Believe & Frais forfaitaires & Autour de $30 \%$ des royalties & $\begin{array}{l}\text { Plus de } 50 \% \text { des royalties (part } \\
\text { plus variable du fait de } \\
\text { contrats moins standardisés) }\end{array}$ \\
\hline $\begin{array}{l}\text { Part dans l'activité } \\
\text { en } 2019\end{array}$ & Environ $20 \%$ & Environ $60 \%$ & Environ $20 \%$ \\
\hline
\end{tabular}

\section{ANALYSE ET DISCUSSION}

Nous commençons par proposer une analyse du modèle de Believe, puis nous mettons en évidence des apports sur les littératures sur l'industrie de la musique à l'ère du numérique et sur le courant BoP.

\subsection{Analyse du modèle de Believe}


Believe a d'abord construit un cœur de métier sur la distribution numérique en développant des outils technologiques très performants. La structure de coûts fixes ainsi que les coûts marginaux quasi nuls d'acquisition de clients, typiques des industries numériques (Varian, Shapiro, 1999), lui ont permis d'adopter des stratégies de volume, dans des métiers qui étaient restés artisanaux, au sens d'une intervention humaine importante. Believe, en s'appuyant sur le numérique, a industrialisé un certain nombre de processus.

La mutualisation constitue ainsi un volet essentiel du modèle de Believe. Le volume permet de mutualiser les coûts de développement des technologies et d'amortir le développement d'outils différenciants pour l'accompagnement des artistes dans l'univers numérique. Pour le volant des artistes traditionnellement dans le giron des maisons de disques, ceux des strates supérieures, Believe combine recours aux technologies numériques et accompagnement artisanal.

Believe couple ainsi une activité de prestataire de service ou offreur de technologies avec une activité éditoriale. La première a vocation à s'adresser au plus grand nombre quand la seconde repose sur une sélection drastique, due notamment aux économies d'échelle très limitées des métiers de l'accompagnement. C'est l'une des originalités du modèle dans un univers où ces deux métiers sont généralement distincts.

Cette combinaison, qui implique une descente dans la chaîne de valeur et une évolution vers les métiers les plus prestigieux, a une autre justification. Le modèle de Believe tire ainsi son originalité de sa capacité à proposer aux artistes un accompagnement à la fois flexible et dans la durée. Au fil d'une carrière classique, l'investissement de l'entreprise est croissant: Believe offre les moyens de base pour se lancer sur le marché puis déploie de nouvelles ressources pour soutenir les succès potentiels. L'articulation des services différenciés et la flexibilité contractuelle permettent à Believe de proposer une offre évolutive qui contraste avec le schéma de «tout ou rien » sur lequel se sont construites les Majors.

La personnalisation des contrats, basée sur le niveau d'engagement des équipes de Believe dans l'accompagnement personnalisé des artistes, permet à l'entreprise de répondre à la variété des besoins des artistes et d'adapter son offre aux dynamiques de carrières et à leur variété. $\mathrm{Si}$, dans le monde physique, le niveau d'implication auprès des artistes dépend de leur succès, l'univers numérique donne lieu à des configurations plus variées, certains artistes à fort niveau de notoriété pouvant prendre en charge leur production et leur promotion. Believe peut conserver dans son giron des artistes qui ré-internalisent ou ont toujours internalisé leur promotion.

Cette capacité à embrasser tout le spectre des situations des artistes a une implication vertueuse. Le volume apporté par le bas de la pyramide et la capacité de fidéliser les artistes les plus porteurs par la souplesse des contrats proposés donne à l'entreprise un pouvoir de négociation notable vis-à-vis de l'autre versant de son marché, les plateformes de distribution, enclenchant un cercle vertueux propre aux marchés bifaces (Rochet et Tirole, 2003) : plus Believe signe d'artistes, plus elle est un partenaire privilégié des plateformes de streaming et, à l'inverse, plus elle entretient un rapport proche avec les plateformes, plus elle devient attractive pour les artistes.

Ce cas met en avant une stratégie de pénétration d'un marché inédite dans les industries créatives qui a été rendue possible par la transformation numérique. Les transformations numériques ouvrent la voie au développement de technologies permettant à l'entreprise de se 
positionner d'abord en tant qu'intermédiaire technique; ce n'est qu'ensuite que sont développées les compétences propres aux industries créatives et à l'industrie musicale. Autrement dit, l'entreprise peut développer un modèle hybride dans le contexte des industries culturelles, où les acteurs choisissent traditionnellement entre un modèle de prestataire technique, n'incluant pas de sélection, basé sur des processus industriels et pouvant être déployé à grande échelle, et un modèle de garde-barrière, assumant une fonction de sélection et une activité à forte teneur artisanale.

\subsection{Apports sur les industries créatives : modèle hybride inédit et restructuration du marché}

Cette analyse permet aussi d'enrichir la compréhension de l'économie des industries culturelles à l'ère numérique. Le numérique a grandement facilité la capacité d'autoproduction des artistes en détruisant les barrières à l'entrée. Si ce phénomène - la désintermédiation et les filières courtes - est resté longtemps théorique (Benghozi, Paris, 2001 ; Hadida, Paris, 2014), le cas Believe et les évolutions qu'il accompagne en donnent une illustration concrète. Ainsi, dans le domaine de la musique urbaine, où les coûts de production peuvent être quasi-nuls, certains artistes se produisent par eux-mêmes, assurent leur promotion et touchent à la consécration sans avoir recours au service d'accompagnement et de promotion offert classiquement par les Majors. Cette réalité montre que la sophistication des outils liés au numérique - pour le ciblage et la promotion notamment - conduit dans certains cas à une séparation de facto des activités de distribution et de prescription/promotion. Les artistes recourent à des prestataires pour les aspects techniques de la distribution dans l'environnement numérique, mais peuvent internaliser les activités de production et de promotion.

Cette situation est susceptible d'ouvrir une nouvelle ère dans les industries créatives qui basculent vers le numérique. Le poids des garde-barrières donnait lieu à une situation qui structurait le marché des artistes en deux catégories - ceux qui avait passé la barrière et les autres (Caves, 2000) - avec une organisation de la chaîne de valeur qui présentait peu de variété : les fonctions artistiques se partageaient entre les labels et les artistes, les fonctions de promotion étaient assurées par les distributeurs. Le numérique, dont l'un des effets escomptés était la segmentation horizontale du marché (Benghozi, Paris, 2001), induit donc un autre effet de segmentation : il casse ce modèle unique et donne lieu à des configurations variées, certains artistes pouvant internaliser - au sein d'entreprises personnelles - la grande majorité des fonctions de production et de promotion, ne faisant appel à des acteurs tiers que pour les fonctions techniques de distribution.

Il en résulte le constat d'une déconnexion entre la classification pyramidale des talents en fonction de leur niveau de notoriété et le niveau de soutien extérieur dont ils disposent. Pendant longtemps, les artistes les plus en vue étaient aussi ceux qui bénéficiaient du plus fort accompagnement. Le cas suggère que des artistes peuvent recourir aux outils développés pour le bas de la pyramide tout en entretenant une notoriété importante.

Ce faisant, ce cas donne aussi une illustration concrète à la théorie de la longue traîne. En couplant prestation technique destinée à tous les artistes et développement d'artistes sélectionnés, une entreprise peut exploiter l'intégralité de la longue traîne en étant rentable. Si le premier argument d'Anderson (2006), affirmant que le numérique allait amener une transformation dans la structuration de consommation n'est pas vérifié, ce cas redonne du 
poids au second argument, à savoir que le numérique permet à des acteurs de générer des modèles rentables sur l'exploitation de la longue traîne.

\subsection{Apports à la littérature BoP : élargir les définitions de bas de la pyramide}

Le concept de stratégie BoP a été développé pour rendre compte des stratégies d'entreprises fondées sur le développement d'une offre pour des consommateurs pauvres. La forme pyramidale qui se dégage de l'organisation de Believe fait écho à la pyramide de consommateurs (ici des artistes) auxquels elle vise à offrir un service (ici la distribution numérique, puis, plus largement, la diffusion). La littérature récente sur les stratégies BoP invite à élargir les définitions premières et les caractéristiques du bas de la pyramide ainsi qu'à caractériser les initiateurs des stratégies BoP afin de questionner les liens entre rentabilité et réduction de la pauvreté (Kolk, River-Santos, Rufin, 2013). Une redéfinition du bas de la pyramide - constitué de populations exclues et non pas de consommateurs pauvres nous permet de mettre en avant des spécificités de ces stratégies et d'enrichir la théorie sur BoP.

\section{Un cas d'exploitation du Bas de la Pyramide}

Les premiers écrits théoriques sur le Bas de la Pyramide suggèrent d'envisager la population ayant un revenu inférieur à $1500 \$$ par an comme une cible rentable (Prahalad, Hart, 2002), pour laquelle les opportunités de marges sont faibles mais les volumes conséquents. Pour des entreprises ayant exploité les strates supérieures de la pyramide, adapter l'offre à ce marché latent permet alors d'élargir la base de consommateurs.

Dans le cas de l'industrie musicale, l'importance des coûts de l'accompagnement personnalisé par les labels de l'ère du disque rendait toute une catégorie d'artistes non-rentables. En développant des outils offrant des solutions peu coûteuses, l'entreprise a modifié cet état de fait et réussi à exploiter ce marché latent. Les moyens novateurs déployés accélèrent les processus, font augmenter les volumes et les économies d'échelle, et au total les coûts baissent drastiquement. Les artistes exclus de l'accès aux ressources de l'industrie deviennent alors des clients d'une offre accessible techniquement et économiquement. Les mécanismes sont, ici, ceux du schéma BoP : la transformation en consommateurs d'un segment jusqu'alors considéré comme non-rentable.

Le cas s'insère également dans la lignée de la deuxième génération d'écrits sur les stratégies BoP, qui élargissent la définition des cibles initialement décrites comme de simples consommateurs finals (Karnani, 2007). En effet, l'artiste n'est plus seulement un fournisseur d'œuvres inséré dans une filière dont la division du travail est linéaire, il n'est plus un travailleur créatif intégré dans une structure intégrée verticalement. Il est à la fois fournisseur d'un produit et client d'un service. Des consommateurs-producteurs qui sont acteurs à part entière de la stratégie BoP.

Alors que la stratégie BoP est le plus souvent développée dans un cadre RSE et que la «base de la pyramide » reste la plupart du temps définie par les consommateurs pauvres, l'industrie de la musique met donc en évidence une autre catégorie de personnes délaissées, celle des artistes-producteurs n'ayant pas accès à un marché et qui constituent un marché latent formant alors le bas de la pyramide de l'industrie musicale. Néanmoins, les mécanismes à l'œuvre sont les mêmes que ceux qui sont mis en évidence dans les travaux $\mathrm{BoP}$, ce qui nous conduit à 
suggérer que la dimension structurante dans les stratégies BoP n'est pas tant constituée par les caractéristiques socio-économiques du marché que sur son volume et son exclusion.

\section{Une stratégie de pénétration plutôt qu'une intention sociale}

Parmi ces consommateurs, la population historiquement écartée du marché - qui constitue le bas de la pyramide de Believe - a été intégrée comme un pilier stratégique de Believe et s'est avérée être un marché profitable. Nous proposons un apport plus générique des stratégies BoP qui dépasse les stratégies d'ouverture à des consommateurs pauvres pour s'intéresser à des marchés latents, délaissés de l'industrie.

Alors que les recherches ont mis en avant des stratégies BoP dans des perspectives de diversification, ce cas suggère que le déploiement d'une stratégie BoP peut sous-tendre une intention de pénétration d'un marché, le marché latent de consommateurs délaissés apparaissant comme un cheval de Troie pour pénétrer un marché verrouillé.

Cela met en avant une séquence chronologique de la stratégie BoP autre que celle mise en avant par les premiers contributeurs de la théorie : alors que ces recherches portaient sur des firmes multinationales qui commençaient par les segments du haut avant de s'attaquer à ceux du bas, nous révélons la faisabilité de la stratégie inverse.

Nous mettons ainsi en évidence une intention stratégique BoP qui relève en premier lieu non pas d'une motivation sociale et environnementale, comme cela est traditionnellement le cas dans le déploiement de ces stratégies (Martinet, Payaud, 2010) mais de l'occasion pour un nouvel entrant de se faire une place dans un marché mature.

\section{CONCLUSION}

Cet article visait à interroger, à travers le cas de l'entreprise Believe dans l'industrie numérique, les conditions de développement d'une stratégie BoP dans un secteur donné.

Nous avons montré que le succès de Believe reposait sur un positionnement qui remettait en cause le fonctionnement du marché de la musique, en s'adressant à un pan exclu des artistes et, ce faisant, en remettant en cause la prééminence des garde-barrières. Alors que cette ouverture avait été annoncée par l'arrivée du numérique avec la notion de longue traîne, elle n'a été exploitée que par le truchement d'une stratégie de pénétration d'un acteur extérieur. Elle a consisté d'abord à se positionner comme intermédiaire technique, alors que le numérique semblait sonner le glas de l'intermédiation. La transformation numérique de la filière a rapidement fait émerger de nouveaux besoins sur lesquels l'entreprise a pu se positionner. Elle a consisté ensuite à répondre aux besoins d'un marché latent d'artistes délaissés par l'industrie, considéré comme non rentable. Le cas est donc celui d'une stratégie opportuniste face à un marché verrouillé, rendue possible par des développements technologiques permettant de traiter un volume important d'artistes, et remettant en cause des schémas considérés comme immuables.

L'ambition de pénétration d'un marché qui a motivé l'entreprise confirme par ailleurs que les stratégies BoP peuvent être envisagées indépendamment d'une mission sociale, ou du moins en en faisant des objectifs secondaires. La construction de l'offre BoP fait également l'objet d'une chronologie inédite: tandis que la littérature met en avant des mouvements d'entreprises établies vers le bas de la pyramide, ce cas rend compte d'une dynamique 
inverse, celle d'une petite entreprise qui pénètre le marché en s'appuyant sur le bas de la pyramide. Le cas invite aussi à adopter une acception plus large de la caractérisation du bas de la pyramide, au-delà du critère de la pauvreté. Elles peuvent être mises en œuvre dès lors qu'un pan de marché est négligé faute de modèle économique viable.

La pérennité de ce modèle est néanmoins suspendue à un environnement concurrentiel aux tensions multiples et qui mettent en cause les avantages construits par Believe : d'une part l'entreprise française n'a pas la maîtrise d'un marché biface dans lequel ses principaux fournisseurs sont des artistes qui contrôlent en grande partie leurs activités, tandis que la diffusion en streaming s'avère être le terrain de jeu des GAFA ; d'autre part, les Majors, en reprenant le modèle de la distribution numérique et des Artists Services, mais aussi en misant sur des actifs économiques exclusifs (la propriété de catalogues en premier lieu), sont parvenues à renouer avec une solide croissance; enfin, les évolutions rapides des usages digitaux rendent plus attractive en même temps qu'elles complexifient la conquête des marchés internationaux. Autant de facteurs d'incertitudes liés à des champs et acteurs qui restent à investiguer plus profondément.

\section{BIBLIOGRAPHIE}

Anderson, C. (2006). The long tail: Why the future of business is selling less of more. Hachette Books.

Anderson, J., \& Billou, N. (2007). Serving the world's poor: innovation at the base of the economic pyramid. Journal of Business Strategy.

Bacache, M., Bourreau, M., Gensollen, M., \& Moreau, F. (2009). Les musiciens dans la révolution numérique. Inquiétude et enthousiasme, Paris, IRMA.

Becker, H. S. (1982). Art Worlds. Univ of California Press.

Becker, H.S. (1998). Les ficelles du métier. La Découverte.

Benghozi, P. J., \& Benhamou, F. (2008). Longue traîne : levier numérique de la diversité culturelle ?. Culture prospective, (1), 1-11.

Benghozi, P. J., \& Paris, T. (2001). L'industrie de la musique à l'âge Internet. Gestion 2000, (2), 41-60.

Benhamou, F. (2002). Économie du star-system (L'). Odile Jacob.

Beuscart, J. S. (2007). Les transformations de l'intermédiation musicale. Réseaux, (2), 143176.

Bourreau, M., \& Labarthe-Piol, B. (2006). Crise des ventes de disques et téléchargements sur les réseaux peer-to-peer. Réseaux, (5), 105-144.

Caves, R. E. (2000). Creative industries: Contracts between art and commerce (No. 20). Harvard University Press.

Cholez, C., Trompette, P., Vinck, D., \& Reverdy, T. (2010). L'exploration des marchés BoP: une entreprise morale. Revue française de gestion, 338, 4551.

DeFillippi, R., Grabher, G., \& Jones, C. (2007). Introduction to paradoxes of creativity: managerial and organizational challenges in the cultural economy. Journal of Organizational Behavior: The International Journal of Industrial, Occupational and Organizational Psychology and Behavior, 28(5), 511-521.

Dumalanède, C. (2017). ONG, initiateurs des stratégies Bottom of the Pyramid ? - Le cas d'Entrepreneurs du Monde. Revue française de gestion, 43(264), 183-204.

Dumez, H. (2016). Méthodologie de la recherche qualitative : Les questions clés de la démarche compréhensive. Vuibert.

Garrette, B., \& Karnani, A. (2010). Challenges in marketing socially useful goods to the poor. California Management Review, 52(4), 29-47. 
Gollakota, K., Gupta, V., \& Bork, J. T. (2010). Reaching customers at the base of the pyramid - a two-stage business strategy. Thunderbird International Business Review, 52(5), 355-367.

Guesalaga, R., \& Marshall, P. (2008). Purchasing power at the bottom of the pyramid: differences across geographic regions and income tiers. Journal of Consumer Marketing, 25(7), 413-418.

Hadida A.L., Paris T. (2014). "Managerial Cognition and the Value Chain in the Digital Music Industry", Technological Forecasting \& Social Change, 83: 84-97.

Karnani, A. (2007). The mirage of marketing to the bottom of the pyramid: How the private sector can help alleviate poverty. California management review, 49(4), 90-111.

Karnani, A.G., Garrette, B., Kassalow, J. S., \& Lee, M. (2010). Better vision for the poor. Ross School of Business Paper, (1137).

Karpik, L. (2007). L'économie des singularités (pp. 12-13). Paris : Gallimard.

Kolk, A., Rivera-Santos, M., \& Rufín, C. (2014). Reviewing a decade of research on the "base/bottom of the pyramid" (BOP) concept. Business \& Society, 53(3), 338-377.

London, T., \& Hart, S. L. (2004). Reinventing strategies for emerging markets: beyond the transnational model. Journal of international business studies, 35(5), 350-370.

Martinet A.C. et Payaud M.A. (2010). « La stratégie BOP à l'épreuve des pauvretés. Une modélisation dialogique », Revue française de gestion, vol. 36, no 208-209, p. 63-81.

Menger, P.-M. (1999): “Artistic Labor Markets and Careers.” Annual Review of Sociology, 25, 541-574.

Moyon, E. (2011). Le changement du business model de l'entreprise: une étude des majors de l'industrie phonographique (1998-2008) (Doctoral dissertation, Lille 1).

Pitta, D., Guesalaga, R., \& Marshall, P. (2008). The quest for the fortune at the bottom of the pyramid: potential and challenges. Journal of consumer marketing.

Prahalad, C. K. (2004). The fortune at the bottom of the pyramid: Eradicating poverty through profits.

Prahalad, C. K., \& Hammond, A. (2002). Serving the world's poor, profitably. Harvard business review, 80(9), 48-59.

Prahalad, C. K., \& Hart, S. L. (2002). The Fortune at the Bottom of the Pyramid in Strategy+ Business, Issue: First Quarter, 2002.

Prahalad, C. K., \& Lieberthal, K. (1998). The end of corporate imperialism. Harvard Business Review, 76(4), 68-80.

Rifkin, J. (2000). L'âge de l'accès : la nouvelle culture du capitalisme. La Découverte.

Rochet, J. C., \& Tirole, J. (2003). Platform competition in two-sided markets. Journal of the european economic association, 1(4), 990-1029.

Schrader, C., Freimann, J., \& Seuring, S. (2012). Business strategy at the base of the pyramid. Business Strategy and the environment, 21(5), 281-298.

Syndicat National de l'Edition Phonographique (2019). L'économie de la production musicale 2019, Edition du SNEP, Neuilly-sur-Seine.

Shapiro, C \& Varian, H.R. (1999). Information Rules. Harvard Business School Press.

Yin, R. K. (2012). Case study methods. 